\title{
One Case of Suspected Angiostenosis Revealed by MRA Following Carotid Endarterectomy Due to a Rare Cause
}

\author{
Yuji Endo ${ }^{*}$, Naoki Sato ${ }^{1}$, Hidekazu Takahashi ${ }^{2}$, Mamoru Ota ${ }^{1}$, Toshihito Ishikawa ${ }^{1}$, \\ Katsuhiro Endo ${ }^{1}$, Kenichi Ebihara ${ }^{1}$
}

${ }^{1}$ Department of Neurosurgery, Masu Memorial Hospital, Fukushima, Japan

${ }^{2}$ Department of Neurosurgery, Ageo Central General Hospital, Saitama, Japan

Email: *yujiendo@masu-med.or.jp

How to cite this paper: Endo, Y., Sato, N., Takahashi, H., Ota, M., Ishikawa, T., Endo, K. and Ebihara, K. (2018) One Case of Suspected Angiostenosis Revealed by MRA Following Carotid Endarterectomy Due to a Rare Cause. Open Journal of Modern Neurosurgery, 8, 276-281.

https://doi.org/10.4236/ojmn.2018.83023

Received: June 13, 2018

Accepted: July 9, 2018

Published: July 12, 2018

Copyright $\odot 2018$ by authors and Scientific Research Publishing Inc. This work is licensed under the Creative Commons Attribution International License (CC BY 4.0).

http://creativecommons.org/licenses/by/4.0/

\begin{abstract}
We herein report on a case in which magnetic resonance angiography (MRA) following carotid endarterectomy revealed the appearance of angiostenosis due to an artifact of metallic powder, which was thought to have come from an old surgical instrument. The patient was a 77-year-old male. Carotid endarterectomy (CEA) was performed for stenosis in the internal carotid artery of his left neck (82\%). Upon observing a decline in renal function following CEA surgery, a postoperative cervical MRA revealed the appearance of high-grade stenosis at the common carotid artery on which surgery was performed. No stenosis was revealed upon carotid ultrasonography and angiography. In the original MRA image, an orbicular low intensity area was observed in concordance with the narrow segment. 3D computed tomography (3D-CT) revealed a metallic finding, suggesting it was an artifact caused by metal powder. Close inspection of the metal surgical instruments used during surgery revealed slight damage to a suture snare which had been used for 30 years. Going forward, it is necessary to pay attention to old surgical instruments, introduce a system by which the number of years and the frequency of use of each surgical instrument can be learned, and replace them with new equipment as necessary.
\end{abstract}

\section{Keywords}

Carotid Endarterectomy, Operation, Metal Artifact, Surgical Instruments

\section{Introduction}

Magnetic Resonance Imaging (MRI) is important for both surgical planning and 
the postoperative follow-up of patients. However, like other imaging techniques, MR imaging is also associated with artifacts, which may related to either the patient or technical causes. It has been reported that postoperative Magnetic Resonance Imaging (MRI) may reveal artifacts due to metallic powder generated when reducing solid bone using a drill during surgery for the spine and acoustic neurinoma [1]-[7]. One widely used form of magnetic resonance angiography (MRA) is the three-dimensional time-of-flight pulse sequence (TOF) method, in which the artery is imaged as a high signal and the image is reconstructed by extracting only the arteries from the original images. This makes it hard to discover the occurrence of artifacts. We hereinafter report on a case in which magnetic resonance angiography (MRA) following carotid endarterectomy revealed the appearance of angiostenosis due to an artifact of metallic powder, which was thought to have come from an old surgical instrument.

\section{Case Report}

The patient was a 77-year-old male with a past history of convulsive seizures and declined renal function (Crea $1.15 \mathrm{mg} / \mathrm{dL}$, normal value: $0.61-1.04 \mathrm{mg} / \mathrm{dL}$ ).

$\mathrm{He}$ developed dysarthria and left hemiplegia. An MRI (diffusion weighted image) revealed a cerebral infarction in the right corona radiata region, while a cervical MRA and three-dimensional computed tomography angiography (3D-CTA) revealed stenosis in the internal carotid artery of his left neck (82\%) (Figure 1). Conservative therapy was carried out but no deterioration of the neurologic manifestation was observed. Carotid endarterectomy (CEA) was performed for stenosis in the internal carotid artery of his left neck.

During the procedure, we used metallic surgical instruments to expose the blood vessels (including a scalpel, bipolar forceps, sucker, dissecting forceps, monopolar, Weitlaner retractor, scissors, forceps, dura elevator, probe, micro needle holder, needle, and subcutaneous drain), as well as temporary clips for cerebral aneurysms in order to block the blood vessels of the superior thyroid artery. When securing vascular access using vascular tape and tourniquet, a suture snare (Mera Heart Suture Holder Rumerushi: SENKO MEDICAL Mfg.

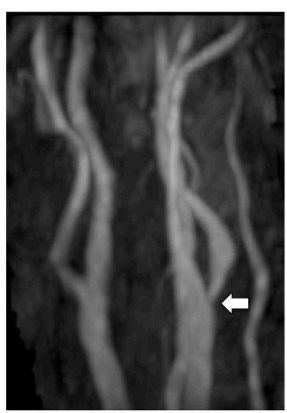

(a)

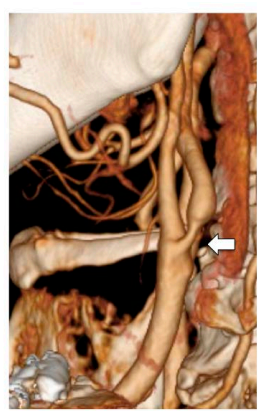

(b)

Figure 1. MRA and 3D-CTA at hospitalization. (a) MRA revealed stenosis in the internal carotid artery of the left neck (arrow); (b) 3D-CTA revealed stenosis in the internal carotid artery of the left neck (arrow). 
Co., Ltd.) was used to insert the vascular tape into the tourniquet. We blocked the blood vessels and carried out surgery as usual using an internal shunt. We completed surgery upon confirmation that there were no problems with the blood stream according to doppler and indocyanine green (ICG) video angiography. No drills potentially resulting in the generation of metallic powder were used.

Postoperative course: While no neurological abnormalities were found following surgery, renal function was found to have declined (Crea $1.24 \mathrm{mg} / \mathrm{dL}$ ). A cervical MRA which had no influence on renal function was performed the next day, revealing high-grade stenosis in the common carotid artery (Figure 2(a)). In the original image, an orbicular low intensity area was observed in concordance with the narrow segment (Figure 2(b)). No narrowing was found upon a carotid ultrasound carried out immediately after. Blood flow velocity was normal. For further detailed investigation, we carried out cerebral angiography using a minimal contrast agent $(10 \mathrm{ml})$ other than $3 \mathrm{D}$-CTA which requires a large amount of contrast agent. No narrowing was found in the common carotid artery (Figure 3(a)). We carried out three-dimensional computed tomography (3D-CT) at the site where the MRA revealed narrowing. There was a slightly high density area at the site, suggesting a finding of metal (Figure $3(\mathrm{~b})$ ). We also tried to image the narrow segment using the spin echo method another day, but were unable to read the image due to a motion artifact from respiration. Upon observation of the metallic surgical instruments used (excluding disposed needles and the puncture site of the subcutaneous drain) using a surgical microscope (ZEISS OPMI ${ }^{\circledast}$ PENTERO $^{\circledR} 900$ ), slight damage was observed at the site of the suture snare through which the vascular tape passes (Figure 4).

The presence of metal powder was explained to the patient, but the patient did not want to undergo further treatment to remove the metal powder. No complications, such as infection occurred during the patient follow-up.

No restenosis occurred during the follow-up.

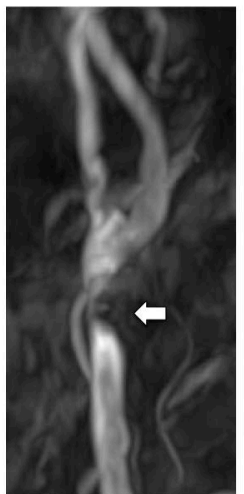

(a)

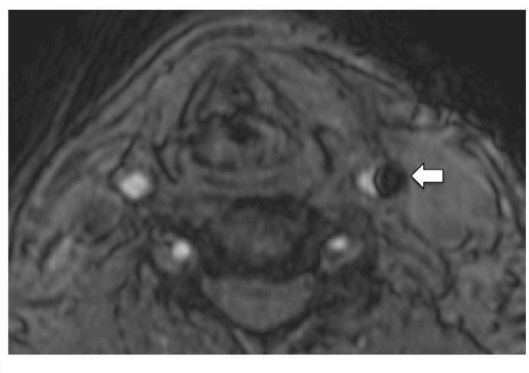

(b)

Figure 2. Postoperative MRA. (a) MRA revealed high-grade stenosis in the left common carotid artery (arrow); (b) in the original MRA image, an orbicular low intensity area was observed in concordance with the narrow segment (arrow). 


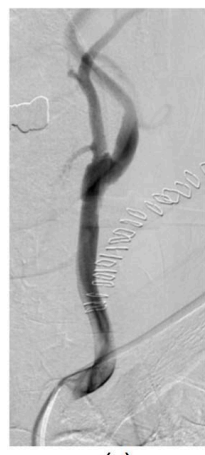

(a)

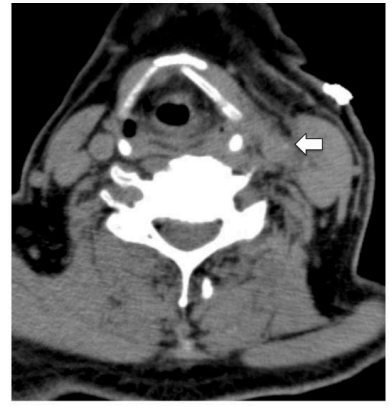

(b)

Figure 3. Postoperative angiography and cervical 3D-CT. (a) Angiography of the left neck did not reveal stenosis in the common carotid artery; (b) the cervical 3D-CT revealed a slightly high density area in the site recognized as stenosis upon MRA (arrow).

(a)

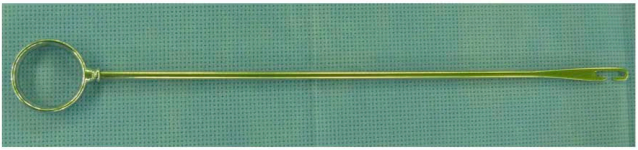

(b)

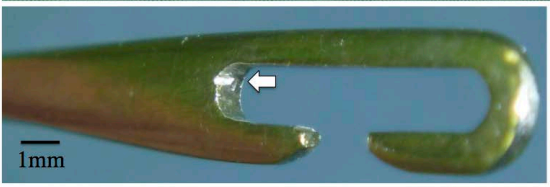

(c)

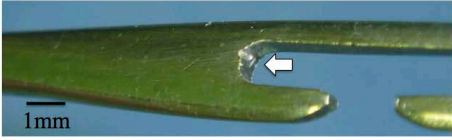

Figure 4. Suture snare. (a) Overall picture; (b) there is damage at the site through which vascular tape passes through the tourniquet (arrow); (c) the damaged part (arrow) is shown from another angle.

\section{Discussion}

This study demonstrated the following two points. First, a cervical MRA following CEA surgery revealed the appearance of high-grade stenosis at the common carotid artery on which surgery was performed, which was thought to be caused by an artifact of metal powder. Second, the artifact of metal powder was thought to be attributed to damage to an old surgical instrument.

Regarding the first point, CT revealed a finding of a metal artifact, suggesting that metal powder was the cause. However, it cannot be completely ruled out that the metal causing the metal artifact in this case may include not only damaged surgical instruments, but also metal powder from the puncture site of the disposed subcutaneous drain, which is attached to the tube, as well as the suture needle. However, in this study, the occurrence site of the artifact coincided with the site at which the common carotid artery was blocked with vascular tape. It is highly likely that vascular tape most closely attached to the same part was involved, with the associated metal surgical instrument thought to be the suture snare that passed the vascular tape through the tourniquet. Because we found a damaged part in the suture snare upon detailed investigation, it can be assumed 
that metal powder from the damage was highly likely to have caused the metal artifact. We assume that fine metal pieces peeled off when passing vascular tape through a tourniquet using a suture snare that had been used for a long time and that the metal powder adhered to the vascular tape then to the blood vessels when the common carotid artery was blocked with vascular tape.

Bertorini et al. found high-grade stenosis in the internal carotid artery upon MRA in patients with a history of CEA. Cerebral angiography revealed that it was pseudo stenosis with no narrowing. They found that the existence of surgical clips used in past surgeries were the cause and reported on the difficulty in noticing the existence of metal artifacts upon MRA based on these facts [8]. The original image should be analyzed to determine whether or not the the stenosis findings upon MRA are artifacts. If metal artifacts are suspected, it is easy and very effective to check by carotid ultrasonography.

Regarding the second point, the cause of damage was thought to be the deterioration of surgical instruments. As far as we are aware, although some have reported the possibility of metallic artifacts due to deteriorating surgical instruments [3], there are no reports indicating that this has actually occurred. According to the manufacturer, the service life of the metal medical equipment was not set and it can be used until damaged. However, the TYPICAL EQUIPMENT LIFETIMES recommended by The American Hospital Association is 10 years for the service life of metal surgical instruments. The period of use of the damaged surgical instrument in this case was 15 years, with 30 years having passed since manufacture. It is difficult to know the period of use of metallic surgical instruments from their appearance if they are well maintained. Furthermore, it was difficult to distinguish the damage to the instrument by the naked eye in this case. The damage was so fine that we had no idea until we suspected damage and observed it using a surgical microscope in detail. Going forward, it is necessary to use a method to efficiently manage all surgical instruments [9] [10] [11], pay sufficient care regarding the use of surgical instruments used longer than their service life, and dispose or replace them as necessary.

Without limiting this to CEA surgery, for cases in which renal function is reduced and contrast agents cannot be used, postoperative MRI and MRA evaluation without using a contrast agent are essential. Deterioration of surgical instruments other than drills may also result in the occurrence of artifacts of metal powder in the surgical field. Even if the metal powder is fine, a significant disadvantage is posed in that MRA cannot be used for follow up thereafter. Therefore, it is necessary to introduce a system to efficiently manage all metal surgical instruments and dispose of surgical instruments used longer than their service life as necessary to protect the safety of patients in the future.

\section{Conclusion}

MRA after CEA revealed a finding of vascular stenosis in the region that had undergone surgery. The cause was thought to be an artifact due to metal powder 
from damage to old surgical instruments. Even if the metal powder is fine, it results in an inaccurate evaluation by MRA, which is a significant disadvantage. Going forward, it is necessary to pay sufficient attention to usable life and service life, etc. for the management of surgical instruments used in the surgical field.

\section{References}

[1] Arunkumar, M.J. and Rajshekhar, V. (1998) Artifacts in Magnetic Resonance Images Following Anterior Cervical Discectomy and Fusion: Report of Two Cases. British Journal of Neurosurgery, 12, 553-555.

[2] Elliott, C.A., Fox, R., Ashforth, R., Gourishankar, S. and Nataraj, A. (2016) Magnetic Resonanceimaging Artifact Following Anterior Cervical Discectomy and Fusion with Atrabecular Metal Cage. Journal of Neurosurgery: Spine, 24, 496-501. https://doi.org/10.3171/2015.5.SPINE14219

[3] Firsching, R.P., Heindel, W., Ernestus, R.I., Frowein, R.A. and Bunke, J. (1987) Postoperativemagnetic Resonance Imaging Artifacts. Report of Three Cases. Journal of Neurosurgery, 67, 776-778. https://doi.org/10.3171/jns.1987.67.5.0776

[4] Peterman, S.B., Hoffman Jr., J.C. and Malko, J.A. (1991) Magnetic Resonance Artifact in the Postoperative Cervical Spine. A Potential Pitfall. Spine (Phila Pa 1976), 16, 721-725.

[5] Sridhar, K., Ramamurthi, R., Vasudevan, M.C., et al. (1999) Magnetic Resonance Imaging Artifact Following Acoustic Neurofibroma Surgery. Case Report. Neurologia Medico-Chirurgica (Tokyo), 39, 938-940. https://doi.org/10.2176/nmc.39.938

[6] Toro, V.E., Goodrich, A., Lundy, D.W., Meeks, L., Figueroa, R.E. and Binet, E.F. (1993) MR Artifacts after Anterior Cervical Diskectomy and Fusion: A Cadaver Study. Journal of Computer Assisted Tomography, 17, 696-699.

https://doi.org/10.1097/00004728-199309000-00005

[7] Yoshino, M.T., Temeltas, O.M., Carter, L.P., Lowe, T. and Darkazanli, A. (1993) Metallicpostoperative Artifacts on Cervical MR. American Journal of Neuroradiology, 14, 747-749.

[8] Bertorini, T.E. and Laster, R. (1995) False Evidence of Carotid Stenosis on Magneticresonance Angiography Caused by Surgical Clips. Journal of Neuroimaging, 5, 206-208. https://doi.org/10.1111/jon199554206

[9] Duro, M. (2014) Surgical Instrument Tracking System Advancements and Benefits. AORN Journal, 100, C7-C8.

[10] Kusuda, K., Yamashita, K., Ohnishi, A., Tanaka, K., Komino, M., Honda, H., Tanaka, S., Okubo, T., Tripette, J. and Ohta, Y. (1988) Management of Surgical Instruments with Radio Frequency Identification Tags. International Journal of Health Care Quality Assurance, 29, 236-247. https://doi.org/10.1108/IJHCQA-03-2015-0034

[11] Yasuhara, H., Fukatsu, K., Komatsu, T., Obayashi, T., Saito, Y. and Uetera, Y. (2012) Prevention of Medical Accidents Caused by Defective Surgical Instruments. Surgery, 151, 153-161. https://doi.org/10.1016/j.surg.2011.06.029 